\title{
A Multi-Format Floating-Point Multiplier for Power-Efficient Operations
}

\author{
Nannarelli, Alberto
}

Published in:

Proceedings of the 30th IEEE International System-on-Chip Conference

Link to article, DOI:

10.1109/SOCC.2017.8226076

Publication date:

2017

Document Version

Peer reviewed version

Link back to DTU Orbit

Citation $(A P A)$ :

Nannarelli, A. (2017). A Multi-Format Floating-Point Multiplier for Power-Efficient Operations. In Proceedings of the 30th IEEE International System-on-Chip Conference (pp. 351-356). IEEE.

https://doi.org/10.1109/SOCC.2017.8226076

\section{General rights}

Copyright and moral rights for the publications made accessible in the public portal are retained by the authors and/or other copyright owners and it is a condition of accessing publications that users recognise and abide by the legal requirements associated with these rights.

- Users may download and print one copy of any publication from the public portal for the purpose of private study or research.

- You may not further distribute the material or use it for any profit-making activity or commercial gain

- You may freely distribute the URL identifying the publication in the public portal

If you believe that this document breaches copyright please contact us providing details, and we will remove access to the work immediately and investigate your claim. 


\title{
A Multi-Format Floating-Point Multiplier for Power-Efficient Operations
}

\author{
Alberto Nannarelli \\ Department of Applied Mathematics and Computer Science \\ Technical University, Denmark \\ alna@dtu.dk
}

\begin{abstract}
In this work, we present a radix-16 multi-format multiplier to multiply 64-bit unsigned integer operands, doubleprecision and single-precision operands. The multiplier is sectioned in two lanes such that two single-precision multiplications can be computed in parallel. Radix-16 is chosen for the reduced number of partial products and the resulting power savings. The experimental results show that high power efficiency is obtained by issuing two single-precision multiplications per cycle. Moreover, by converting the double-precision numbers which fit to single-precision, further energy can be saved.
\end{abstract}

\section{INTRODUCTION}

Several computing applications require high throughput (parallel) multiplications. Multiplication is a very power demanding operation and increasing its efficiency is highly desirable especially in systems performing several multiplications per cycle in parallel, such as accelerators, multi-lane vector units and GPUs.

Current implementations of parallel binary multiplication $\mathrm{X} \times \mathrm{Y}$ follow the steps of [1]: 1) recoding of the multiplier $Y, 2)$ digit multiplication of each digit by the multiplicand $X$, resulting in a number of partial products (PPs), 3) reduction of the partial product array to two operands using multioperand addition techniques, and 4) carry-propagate addition of the two operands to obtain the final result.

What makes multiplication power hungry is the large adder tree to reduce the partial products (PPs) to the final product, especially if the operands have a large number of bits [2]. By resorting to a higher radix, the number of PPs can be reduced.

A very popular scheme for parallel multiplication is radix-4, where for a 32-bit multiplier Y, the PPs are 32/2=16 [3]. As the wordlength becomes longer, the radix has been extended to radix-8 [4]-[6] and even radix-16 [7]. The advantage is that the PP reduction tree is shallower (faster and less power demanding) at expenses of a more complicate PP generation. For radix-8, $3 \times \mathrm{X}$ must be computed and this requires a carrypropagate addition: $3 X=2 X+1$. However, floating-point (FP) multipliers are normally pipelined, and if the latency of the pre-computation can be contained in a single pipeline stage, high radix multipliers become attractive because we can save power.

In this work, we present a radix-16 multiplier supporting both integer and FP multiplication in binary32 (single precision) and binary64 (double precision). Moreover, the multiplier can execute two binary 32 multiplications in parallel (dual lane). We chose radix-16 to limit the depth of the PP accumulation tree and save power. The unit was inspired by the implementation in the Intel Itanium FP-unit [7].

The main contributions of this paper are:

- A trade-off analysis of the power dissipation between the radix-4 and radix-16 implementation for a $64 \times 64$ multiplier (Sec. II).

- A pipelined implementation of the radix-64 multi-format multiplier with a power analysis for the different formats (Sec. III).

- We propose a simple method to reduce from double to single precision FP numbers to save power (Sec. IV).

\section{BASEline RADiX-16 MultiplieR}

In this section, we briefly describe the architecture of the basic radix-16 multiplier for 64-bit unsigned operands. We denote $\mathrm{X}$ the multiplicand operand and $\mathrm{Y}$ the multiplier.

The first multiplication step is the recoding of the multiplier operand Y: groups of four bits $\left(0 \leq Y_{i} \leq 15\right)$ are recoded in the minimally redundant radix-16 digit set $\{-8,-7, \ldots, 0, \ldots, 7,8\}$. This recoding is done carry-free and the transfer-digit (see [1] for detail) is the most-significant bit (MSB) of the four-bit group $Y_{i}$.

Because of the radix-16 recoding, the number of partial products (PPs) is 16 for a 64-bit Y. However, due to a possible transfer digit from the most significant radix-16 digit, the number of resultant radix-16 recoded digits is $\lceil(n+1) / 4\rceil$ for the general case of a $n$-bit multiplier operand. Therefore, for $n=64$ the number of PPs is 17 . The most significant PP, is either 0 or $X$ shifted 64 bits to the left (16 radix-16, 4 bits, positions). A method to reduce the number of PPs from 17 to 16 is presented in [8].

In parallel with the recoding, the odd multiples of $\mathrm{X}(3 \times \mathrm{X}$, $5 \times X$, and $7 \times X)$ are precomputed by three fast carry-propagate adders (CPAs), implementing $\mathrm{X}+2 \mathrm{X}=3 \mathrm{X}, \mathrm{X}+4 \mathrm{X}=5 \mathrm{X}$, and $8 \mathrm{X}+\mathrm{X}=7 \mathrm{X}$, respectively. The values $2 \mathrm{X}, 4 \mathrm{X}$ and $8 \mathrm{X}$ are obtained by simple wiring (left shifts) of $\mathrm{X}$. Moreover, 6X is obtained by a simple one bit left shift of $3 \mathrm{X}$.

The PPs are then generated by selecting a multiple of $\mathrm{X}$ depending on the value of the radix-16 digit $Y_{i}$. Fig. 1 illustrates a possible implementation of the PPs generation. An array of XOR gates, complements the bits of the PP when the recoded multiplier digit is negative. 


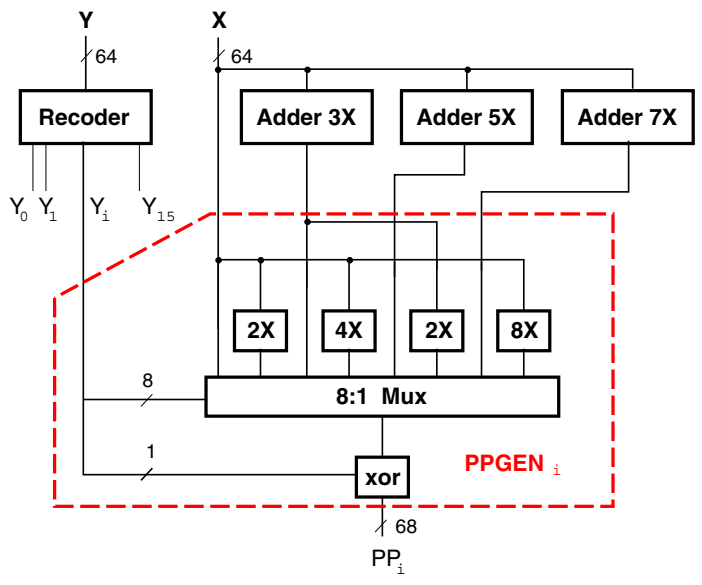

Fig. 1. Partial product generation.

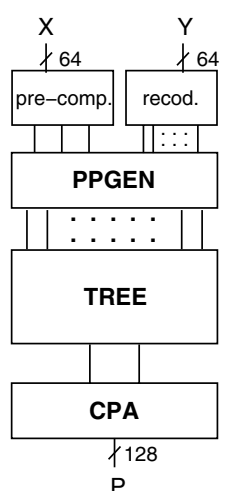

Fig. 2. Radix-16 multiplier.

Each partial product has $n+4$ bits ( 68 bits) including the sign in two's complement representation. The extra four bits are required for the digit multiplication by up to 8 and the sign bit.

The costly sign extension in the PP array is avoided by the standard method of sign extension reduction and correction [1].

After the generation of the partial product bit array, the reduction (multioperand addition) from a maximum height of 17 (for $n=64$ ) to 2 is implemented by $3: 2$ or $4: 2$ carry-save adders (CSAs).

After the reduction to two operands, a carry-propagate addition is performed. The complete block diagram for radix16 multiplication is depicted in Fig. 2.

The baseline $64 \times 64$ radix- 16 multiplier is implemented in a $45 \mathrm{~nm}$ CMOS library of standard cells by using commercial synthesis tools. The FO4 delay for this low power library is $64 \mathrm{ps}$ and the area of the NAND-2 gate is $1.06 \mu \mathrm{m}^{2}$.

Referring to Fig. 1 and Fig. 2, the delay of the critical path is reported in Table I along with the multiplier's area.

\begin{tabular}{ccccc}
\hline Critical Path & & & & \\
\hline pre-comput. & PPGEN & TREE & CPA \\
578 & 258 & 571 & $445=1852 \mathrm{ps}$ \\
\hline
\end{tabular}

\begin{tabular}{cccc}
\hline \multicolumn{2}{c}{ Latency } & \multicolumn{2}{c}{ Area } \\
{$[n s]$} & FO4 & {$\left[\mu m^{2}\right]$} & NAND2 \\
\hline 1.852 & 29 & 50,562 & $47.8 \mathrm{~K}$ \\
\hline
\end{tabular}

TABLE I

LATENCY, AREA AND CRITICAL PATH OF 64×64 RADIX-16 MULTIPLIER.

\begin{tabular}{|c|c|c|c|}
\hline \multicolumn{4}{|l|}{ Critical Path } \\
\hline PPGEN & TREE & CPA & \\
\hline 313 & 739 & 454 & $1506 p s$ \\
\hline Latency & & \multicolumn{2}{|c|}{ Area } \\
\hline$[n s] \quad$ FO4 & & {$\left[\mu m^{2}\right]$} & NAND2 \\
\hline 1.506 & & 60,204 & $56.9 \mathrm{~K}$ \\
\hline
\end{tabular}

TABLE II

LATENCY, AREA AND CRITICAL PATH OF 64×64 RADIX-4 MULTIPLIER.

\section{A. Comparison Radix-4 vs. Radix-16 Multiplication}

The main problem with high radices is the computation time of the odd multiples (pre-computation in Table I). However, since FP multipliers are normally pipelined, if the precomputation can be accommodated in a pipeline stage, its delay is not critical.

To confirm that the radix-16 multiplier consumes less energy than the common radix- 4 multiplier, we implement a $64 \times 64$ radix-4 Booth multiplier.

We do not implement a radix-8 multiplier because it also needs the pre-computation of $3 \mathrm{X}$, but its reduction tree is larger than the radix-16 tree.

The result of the radix- 4 implementation are reported in Table II.

By comparing Table I and Table II, as expected, the radix-4 unit is about $20 \%$ faster than the radix-16 unit, but, due to the larger tree the radix-4 unit area is about $18 \%$ larger.

The power dissipation for the two units is reported in Table III. The first row refers to the combinational implementation, the second row refers to a two-stage pipelined implementation. All power estimates are done at clock frequency $100 \mathrm{MHz}$. Note that for the pipelined version of the multiplier, both implementations have the same latency of 2 clock cycles.

For both implementations the radix-16 consumes less power than the radix -4 . The radix- 16 has a longer delay, therefore, in the combinational implementation the power due to glitches is probably larger than in the radix-4 unit. In the pipelined implementation, the paths in the two stages are shallower and the glitching power is reduced. As a result, the power savings in the radix-16 multiplier increase from $6 \%$ to $11 \%$. 


\begin{tabular}{lccc}
\hline & \multicolumn{2}{c}{ Power $[m W]$} & \\
& radix-4 & radix-16 & Ratio \\
\hline Combinational & 12.3 & 11.5 & 0.94 \\
two-stage pipelined & 8.7 & 7.7 & 0.89 \\
\hline
\end{tabular}

TABLE III

POWER DISSIPATION AT 100 MHZ FOR RADIX-4 AND RADIX-16 MULTIPLIERS.

\begin{tabular}{|lrrrr|}
\hline & \multicolumn{4}{c|}{ binary } \\
Format & 16 & 32 & 64 & 128 \\
\hline Storage (bits) & 16 & 32 & 64 & 128 \\
Precision $f=1$ (bits) & 11 & 24 & 53 & 113 \\
Total exponent length (bits) & 5 & 8 & 11 & 15 \\
$E_{\max }$ & 15 & 127 & 1023 & 16383 \\
bias & 15 & 127 & 1023 & 16383 \\
Trailing significand $f$ (bits) & 10 & 23 & 52 & 112 \\
\hline
\end{tabular}

TABLE IV

BINARY FORMATS IN IEEE 754-2008 [9].

\section{Multi-Format FP-Multiplier}

The starting point to build the Multi-Format FP-multiplier (MFmult) is the radix-16 $64 \times 64$ multiplier of Sec. II. The MFmult should support the following formats:

- int64: multiplication of two 64-bit unsigned integers producing a 128-bit product. This is the operation implemented by the unit of Sec. II.

- fp64: multiplication of two floating-point binary64 numbers (formerly, double-precision), producing a binary64 result.

- fp32: two multiplications of two floating-point binary32 numbers (single-precision), producing two binary 32 results.

The int64 format provides a 128 product that can be used for ad-hoc operations in extended precision.

To accommodate the FP multiplications, the unit of Fig. 2 must be augmented by normalization and rounding hardware and sign and exponent handling. The formats for binary 32 and binary64 are specified in Table IV [9].

\section{A. Multiplication binary64}

The binary64 FP format requires 64 bits of storage: 1 bit for the sign, 11 bits for the exponent, and 52 bits for the fractional part of the significand. If the biased exponent is larger than zero, the integer bit is ' 1 ' and the resulting significand is 53 bits. Therefore, the $53 \times 53$ significand multiplication can easily be accommodated in the $64 \times 64$ multiplier of Fig. 2 by aligning the operands $\mathrm{X}$ and $\mathrm{Y}$ to the least-significant bit of $64 \times 64$ multiplication.

Once the product is computed, the result may not be normalized [1]. For the multiplication of normalized numbers the leading ' 1 ' could be either in bit 105 or in bit 104 (bit 0 is the LSB). Consequently, a 2:1 multiplexer is required to shift the product one position to the left if the leading ' 1 ' is in bit 104.

Afterwards, we need to perform the rounding by adding ' 1 ' in position 52 . By truncating the result in position 53 , we

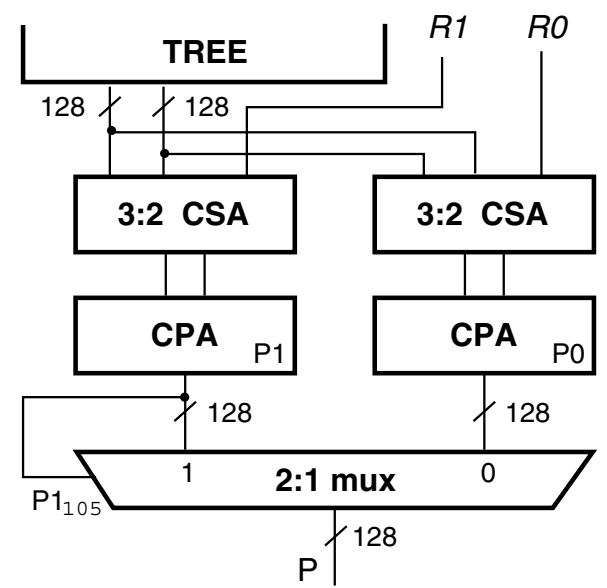

Fig. 3. Detail of the normalize and round operations.

obtain the 53 bits $\left(P_{105} \ldots P_{53}\right)$ of the normalized and rounded significand.

Since the rounding step requires a carry-propagate addition, expensive in terms of latency, we can speculatively compute the rounded product for both cases in which the leading ' 1 ' is in position 105 or 104 , and use the normalization mux to select the normalized product. This is illustrated in Fig. 3. In the figure, $R 1$ injects a ' 1 ' in position $53\left(0 \ldots 001_{53} 00 \ldots 0_{0}\right)$, $R 0$ injects a ' 1 ' in position $52\left(0 \ldots 0001_{52} 0 \ldots 0_{0}\right)$.

This combined normalization and rounding requires an extra fast CPA and extra gates to implement the CSAs ${ }^{1}$.

Clearly, for int64 multiplications, only one CPA is used, $R 1$ and $R 0$ are set to zero, and the mux-select is set to ' 0 ' regardless of bit 105 of P1 (the control for this case is not depicted in Fig. 3).

Currently, the binary64 multiplier does not support rounding to the nearest in case of a tie (no sticky bit computation) and rounding of subnormals.

\section{B. Two Multiplications binary 32}

The binary $32 \mathrm{FP}$ format requires 32 bits of storage: 1 bit for the sign, 8 bits for the exponent, and 23 bits for the fractional significand (24 bits for the normalized significand).

We can accommodate two binary32 multiplications ( $X Y$ and $W Z$ ) in the $64 \times 64$ multiplier. The operands of the multiplication $X Y$ are aligned to bit in position 0 (LSB) and the operands of $W Z$ to bit 32 . The arrangement in the PP array is sketched in Fig. 4. The lower part of the array computes $X Y$, the upper part $W Z$.

The PPGEN block must be modified to "blank" bits of the PP and allow a correct carry-propagation and sign-extension correction for the two independent multiplications. The two CPAs and the mux/shifter of Fig. 3 are divided in an upper and lower part, and the shifter control is separated for the two parts. For example, the upper significand is not shifted while the lower significand is shifted one position to the left.

\footnotetext{
${ }^{1}$ Since $R 1$ and $R 0$ contains only one non-zero bit, the CSA is made of one full-adder and 74 half-adders.
} 


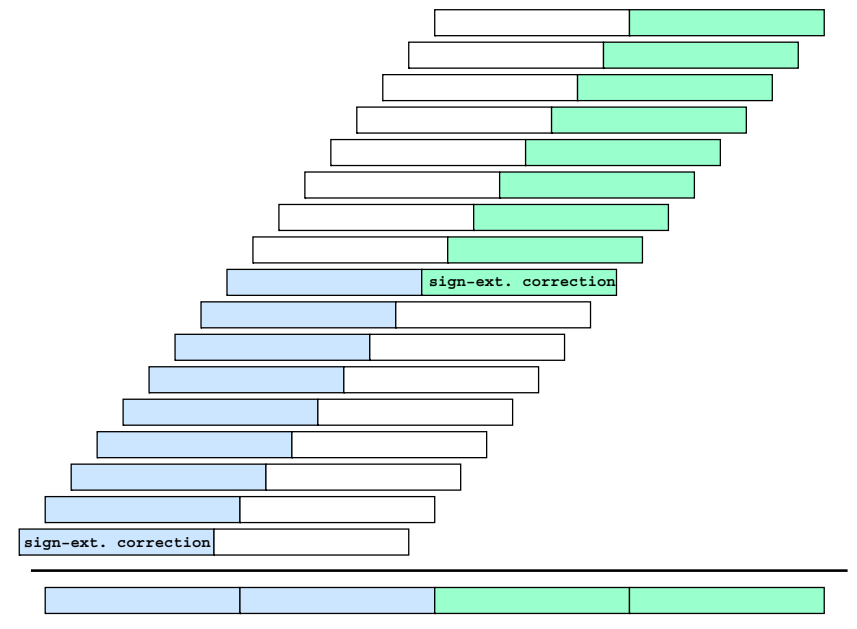

Fig. 4. Array arrangement for two binary32 multiplications.

Moreover, the injection vectors used for rounding, $R 1$ and $R 0$, are modified to round in the upper and lower parts of the array:

$R 1=0 \ldots 01_{87} 00 \ldots 0_{64} \ldots 01_{23} 00 \ldots 0_{0}$

$R 0=0 \ldots 001_{86} 0 \ldots 064 \ldots 001_{22} 0 \ldots 0_{0}$.

\section{Sign and Exponent Handling}

Sign and exponent handling (S\&EH) is quite straightforward for FP multiplication. For a single multiplication, the sign of the product is the XOR of the sign of the two operands, and the exponent is computed as follows:

1) sum of the two exponents $E_{X}, E_{Y}$ diminished by the bias $B: E_{P}=E_{X}+E_{Y}-B$;

2) $E_{P}$ is incremented by 1, if the leading ' 1 ' of the significand is in bit position 105: $E_{P}=E_{P}+1$.

For dual binary32 multiplication, the $S \& E H$ must be duplicated. The exponent handling of the upper (in the array) multiplication is shared by the binary64 and the binary 32 operations (11-bit adders), while for the lower binary32 multiplication a 8-bit datapath is used.

\section{Pipelined Unit}

The different components to support the three formats are put together next.

We chose as a target clock period the delay of 16 FO4, corresponding to about $1 \mathrm{~ns}$ in our library, to get a throughput of about 1 GFLOPS for binary64. With this choice, we pipeline the unit in three stages by placing pipeline stages as indicated in Fig. 5. The figure does not show the replicated hardware for the S\&EH of the lower binary32 multiplication to simplify the drawing. Moreover, control signals to differentiate among the three formats are omitted as well in Fig. 5.

The block input formatter in Fig. 5 transfers the operands' bits to the functional units in stage- 1 depending on the format specified in frmt.

Similarly, the block output formatter transfers the result bits to the output. The unit output is split in two 64-bit

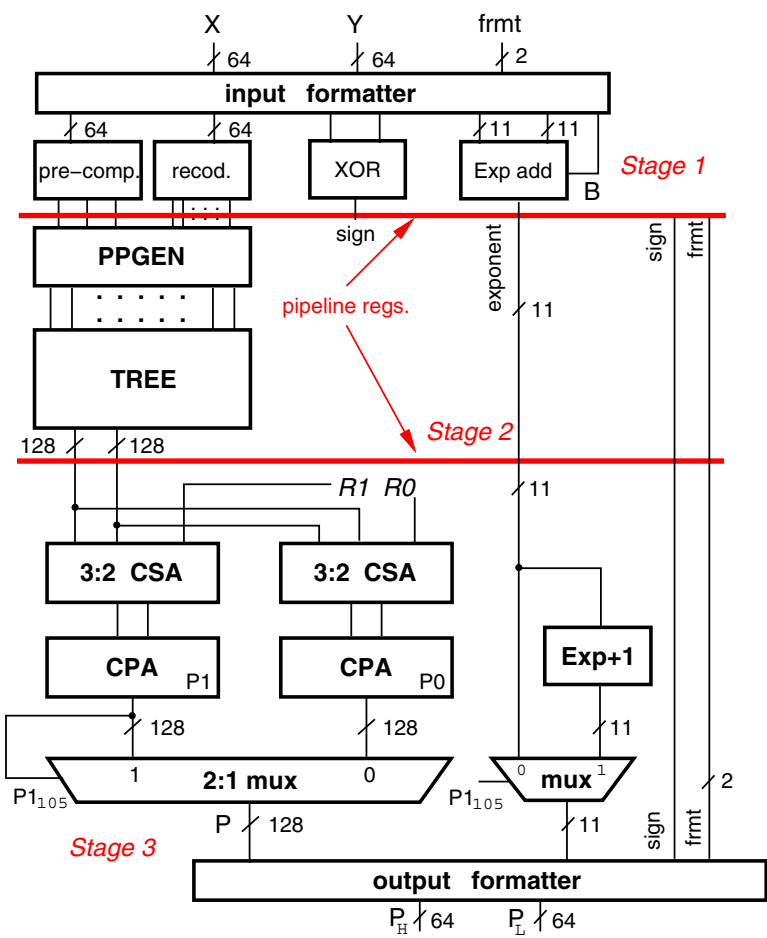

Fig. 5. Pipelined implementation of the multi-format multiplier.

parts $P_{H}$ and $P_{L}$. For int64 multiplications, the 128-bit product is output on both ports. For binary 64 and binary 32 multiplications, the output $P_{L}$ is not used. For dual binary 32 multiplication, one product is placed in the $32 \mathrm{MSBs}$ of $P_{H}$, and the other product in the $32 \mathrm{LSBs}$ of $P_{H}$.

To save time, the exponent is incremented speculatively in stage-3, and then the right exponent is selected once $P 1_{105}$ is determined (binary64).

For binary32, the normalization and the exponent selection is done according to $P 1_{111}$ for the upper multiplication, and to $P 1_{47}$ for the lower multiplication. This is not depicted in Fig. 5 to keep the drawing as simple as possible.

The pipelined unit is synthesized in the $45 \mathrm{~nm}$ library. The resulting critical path of $1120 \mathrm{ps}$ is in stage-2. This corresponds to about 17.5 FO4 delay, 1.5 FO4 in excess of the target cycle time. The pipeline overhead (pipeline register delay and set-up time) is about 3 FO4 (depending on the register loading). According to Table I, the combinational delay in stage-2 (PPGEN+TREE) is about 830 ps or 13 FO4, however, the insertion of the pipeline registers altered the slacks and increased the stage delay.

We tried to move the pipeline registers after the PPGEN, but then the critical path moved in stage- 1 . We also tried to move the pipeline registers between stage- 2 and stage- 3 , by placing registers inside TREE, but then stage- 3 became critical. Since the improvements in the timing are marginal, we settle with the pipeline placement of Fig. 5 which is the one with the lowest number of pipeline registers among the tried placements. 


\begin{tabular}{lcccc}
\hline \multirow{2}{*}{ Format } & \multicolumn{2}{c}{ Power $[\mathrm{mW}]$} & throughput & $\begin{array}{c}\text { Power eff. } \\
\text { [GFLOPS/W] }\end{array}$ \\
\hline int64 & $100 \mathrm{MHz}$ & $880 \mathrm{MHz}$ & [GFLOPS] & [G.32 \\
binary64 & 8.90 & 0.88 & 11.24 \\
binary32 (dual) & 5.17 & 45.50 & 1.76 & 38.68 \\
binary32 (single) & 3.77 & 33.18 & 0.88 & 26.53 \\
\hline
\end{tabular}

TABLE V

POWER DISSIPATION AND POWER EFFICIENCY FOR THE DIFFERENT FORMATS.

\section{E. Power Efficiency}

Next we estimate the power dissipation for the different multiplication formats. We perform a Monte Carlo simulation by generating pseudo-random input patterns and estimate the power at a reference frequency $100 \mathrm{MHz}$ to have an easily scalable value to any frequency: our design can be clocked at $880 \mathrm{MHz}$ maximum.

Table $\mathrm{V}$ reports the results of the power estimation for the different formats. The throughput is computed as one multiplication per clock cycle for int64 and binary 64 and two multiplications per cycle for dual binary32. Moreover, we report the power dissipation when only one binary 32 operation is issued in the multiplier.

The rightmost column in Table $\mathrm{V}$ lists the power efficiency expressed as FLOPS per watt.

The different values of power dissipation in the table are due to different activity in the multiplier. With respect to the int 64 , when a binary 64 multiplication is executed only $\frac{53 \times 53}{64 \times 64}=68 \%$ of the bits in the significand datapath are meaningful. The power dissipation ratio binary64/int64 in Table $\mathrm{V}$ is about $80 \%$. There is clearly some $10 \%$ overhead due to the activity in the S\&EH that is inactive for int64 operations.

As for the power efficiency, the extra efficiency in binary64 over the int64 is due to the lower dissipation. For binary 32 we obtain the best efficiency because we complete two multiplications per clock cycle and spend less power than binary64. By issuing only one binary32 multiplication per clock cycle, we double the power efficiency with respect to binary64 at expenses of a lower precision.

\section{Improved Multi-Format FP-Multiplier}

The results for power efficiency of Table $\mathrm{V}$ suggest to use binary32 FP format, if the application allows for a reduced precision. This is probably the case for many applications, for example, multiplication of small integers or small fractions.

Since even a single binary 32 is more power efficient than binary 64 , by reducing the precision of operands, we can always save power.

Next, we propose a simple method to convert error-free a binary64 FP number in a binary32 FP number, when the non-zero bits of the binary64 significand can be represented by a binary32 significand (i.e., number of nonzero bits $\leq 23$ ), and the range is representable (i.e., unbiased exponent $[-127,127])$.

The algorithm is shown in Algorithm 1 and its hardware architecture is sketched in Fig. 6.
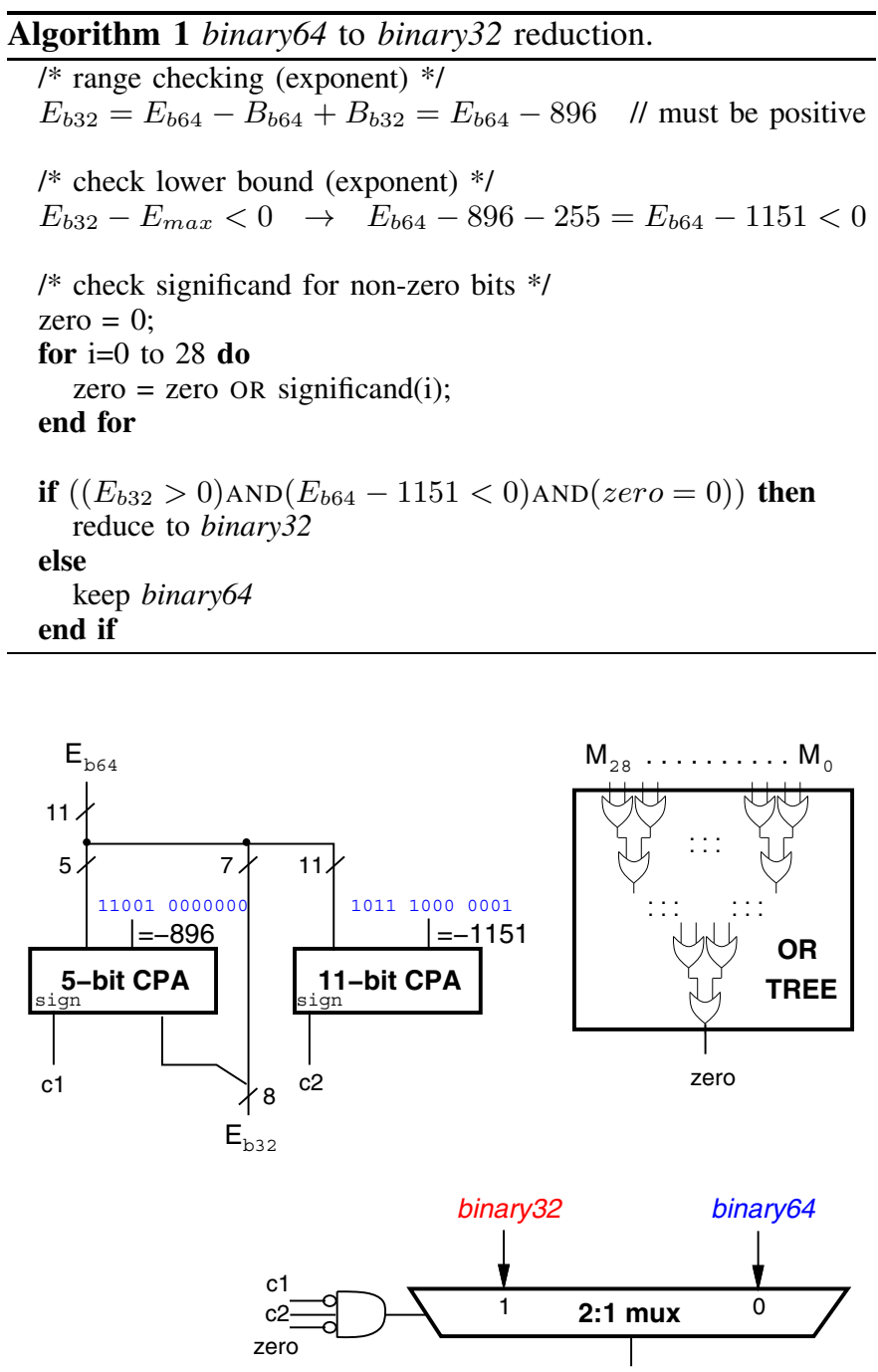

Fig. 6. Hardware for binary64 to binary32 reduction.

The reduction of the exponent (first statement in Algorithm 1) can be implemented by a 5-bit adder since the 7 LSBs of -896 are zero. In contrast, to check the lower bound (second statement in Algorithm 1) a 12-bit adder is needed since -1151 is odd.

To check whether the 29 LSBs of the significand ( $M$ in Fig. 6) are zero ("for" loop in Algorithm 1), we can use a tree of OR gates. The selection is done with a multiplexer based on the MSB (sign bit) of $E_{b 32}$ and $E_{b 64}-1151$, and on the output of the OR-tree. Clearly, the sign bit of the binary 64 is transferred to the binary32 FP number.

The small hardware of Fig. 6 can be easily included in the multi-format multiplier of Fig. 5. The two short additions can be done in parallel with the speculative exponent computation. Part of the OR-tree can be shared with the sticky-bit computation (not yet implemented in Fig. 5). The selection between binary 32 (reduced) or binary 64 can be easily accommodated in the output formatter block. 


\section{CONCLUSIONS AND Future Work}

Radix-16 multiplication is an attractive alternative to implement the product of operands with large wordlength because of the reduced PP accumulation tree, which results is smaller silicon area and lower power dissipation.

Another advantage of a reduced number of PPs is that it makes easier the sectioning of the PP array to perform multilane operations on operands of reduced wordlength.

We took advantage of the radix-16 scheme to implement a multi-format multiplier. The power analysis on the implemented unit shows that we can increase the power efficiency with respect to binary64 operations by executing one or two binary32 multiplications.

Therefore, the proposed unit provides the flexibility of three formats, depending on the application, and power efficiency when the dynamic range of the operands (precision) can be traded-off for power dissipation.

In future work, we plan to extend the reduction binary64 to binary 32 to periodic numbers as well, and incorporate the reduction in the multi-format unit.

\section{REFERENCES}

[1] M. Ercegovac and T. Lang, Digital Arithmetic. Morgan Kaufmann Publishers, 2004

[2] S.Galal et al., "FPU Generator for Design Space Exploration," in Proc. 21st IEEE Symposium on Computer Arithmetic (ARITH), Apr. 2013, pp. $25-34$.

[3] K. Tsoumanis et al., "An Optimized Modified Booth Recoder for Efficient Design of the Add-Multiply Operator," IEEE Transactions on Circuits and Systems I: Regular Papers, vol. 61, no. 4, pp. 1133-1143, Apr. 2014.

[4] E. M. Schwarz, R. M. A. III, and L. J. Sigal, "A radix-8 CMOS S/390 multiplier," in Proc. 13th IEEE Symposium on Computer Arithmetic (ARITH), July 1997, pp. 2-9.

[5] S. Oberman, "Floating point division and square root algorithms and implementation in the AMD-K7 microprocessor," in Proc. 14th IEEE Symposium on Computer Arithmetic (ARITH), Apr. 1999, pp. 106-115.

[6] G. Colon-Bonet and P. Winterrowd, "Multiplier Evolution: A Family of Multiplier VLSI Implementations," The Computer Journal, vol. 51, no. 5, pp. 585-594, 2008

[7] R. Riedlinger et al., "A $32 \mathrm{~nm}, 3.1$ Billion Transistor, 12 Wide Issue Itanium Processor for Mission-Critical Servers," IEEE Journal of SolidState Circuits, vol. 47, no. 1, pp. 177-193, Jan. 2012.

[8] E. Antelo, P. Montuschi, and A. Nannarelli, "Improved 64-bit Radix-16 Booth Multiplier Based on Partial Product Array Height Reduction," IEEE Transactions on Circuits and Systems I, vol. 64, no. 2, pp. 409-418, Feb. 2017.

[9] IEEE Standard for Floating-Point Arithmetic, IEEE Computer Society Std. 754, 2008 